\title{
Un modelo de evaluación de las revistas científicas con sus más y sus menos
}

El Departamento Administrativo de Ciencia, Tecnología e Innovación - Colciencias, a través del nuevo modelo de clasificación de revistas científicas, intenta promover una divulgación que cumpla realmente con el intercambio de conocimiento entre la comunidad científica, lo cual actualmente no es un logro de la mayoría de las revistas colombianas. Según datos revelados en el documento definitivo de Publindex (https://goo.gl/8hnMds), solo el 13\% de las revistas científicas colombianas se encuentran incluidas dentro de índices bibliográficos citacionales y su índice citacional es de 3; muy por debajo del promedio en Latinoamérica. El índice $h$ y más concretamente el $h 5$, el cual fue utilizado por Colciencias para este fin, consiste en el cálculo del número de citaciones recibidas por una publicación científica en los últimos cinco años. El dato de 3 para Colombia da cuenta de dos aspectos según el documento de Publindex. Uno de ellos es la baja producción científica de los investigadores colombianos, junto con su bajo impacto; sumado a la limitada visibilidad y el bajo impacto de las revistas colombianas.

En miras a cumplir con altos estándares de calidad, es importante medir nuestra visibilidad e impacto científico, de la misma manera como se mide en la mayoría de los países generadores de conocimiento de gran trascendencia. Es por esto que el nuevo modelo impulsa a la realización de un trabajo de calidad, organizado y sin escatimar que somos un país tercermundista; pero, además, es coherente con las diferentes dinámicas en el interior de distintas disciplinas y, por tanto, involucra una valoración diferenciada del impacto en las áreas del conocimiento.

El nuevo modelo tuvo presente criterios relacionados con la gestión editorial, la visibilidad y el impacto, y determinó tres fases de validación, con el objeto de clasificar de manera adecuada a las revistas científicas colombianas:

Fase I: Declaración de cumplimiento del proceso de gestión editorial.

Fase II: Validación del proceso de evaluación y visibilidad.

Fase III: Impacto de las revistas científicas.

La primera fase contempla doce criterios, los cuales deben ser cumplidos en su totalidad para poder pasar a la fase siguiente. Si bien en esta parte se establecen criterios que contribuyen con el incremento de la calidad de las revistas científicas, uno de los aspectos más innovadores es el requisito de la «accesibilidad». En este punto se aclara la importancia de disponer los contenidos en línea, apoyando la publicación de revistas electrónicas por encima de aquellas que tradicionalmente se han producido exclusivamente en formato impreso. Esto sumado a que el sitio web de la revista debe contener la información tanto en español como en inglés, favoreciendo la visibilidad e impacto de las publicaciones científicas.

En la fase II básicamente el nuevo modelo se preocupa por la conformación del comité editorial y científico de las revistas, así como por la internacionalización de la misma y los criterios de calidad implementados para el proceso de evaluación, ya que esto asegura mejores prácticas editoriales. En este punto cabe resaltar la «visibilidad», condición indispensable para lograr el cometido final de las ciencias: la transmisión del conocimiento, lo que a su vez genera más conocimiento y trabajo en redes.

Finalmente, en la fase III se contempla el impacto de las revistas científicas, incluyendo su posición de acuerdo con las herramientas tradicionales que evalúan este criterio, como el JCR (Journal Citation Report) y SJR (Scimago Journal Rank). Sin embargo, también contempla la visibilidad científica de las revistas teniendo como fuente el Google Scholar, mediante el cálculo del índice h5, con el cual se evalúa el impacto de la revista científica contemplando el número de citas por artículo en un período de cinco años. Esta propuesta interesante e innovadora trae 


\section{RHS Revista Humanismo \\ RHS y sociedad}

consigo retos para las revistas y para los investigadores y sugiere una serie de medidas que deben ser tomadas por estos en aras de fomentar la visibilidad de su producción científica. Esto incluye no remitirse solo a la publicación, sino, además, encargarse y volverse protagonista de la divulgación de este material en diferentes redes del conocimiento, incluyendo el Google Scholar. Este tipo de prácticas son importantes, e independiente del nuevo modelo de Publindex, deberían ser consideradas por todas las revistas científicas, ya que constituyen una innovadora y correcta práctica editorial.

Lina M. Yassin

Doctora en Ciencias Básicas Biomédicas

Vicerrectora de Investigaciones

Corporación Universitaria Remington 